\title{
A UTILIZAÇÃO DO SISTEMA R-STUDIO E DA JURIMETRIA COMO FERRAMENTAS COMPLEMENTARES A PESQUISA JURÍDICA
}

\author{
Mariana Dionísio de Andrade ${ }^{1}$
}

\section{Resumo}

O presente trabalho possui como objetivo responder ao seguinte questionamento: é possível construir uma ponte dialógica e didática entre a pesquisa jurídica de alto nível e a utilização de métodos com base econométrica? A ideia central se constitui na utilização do software R-Studio, típico de estudos estatísticos, como ferramenta de apoio à pesquisa jurídica com base em análise de dados, o que pode já é realizado pela jurimetria e deve ser amplamente difundido por práticas docentes. É essencial que haja complementaridade entre a pesquisa quantitativa e qualitativa, justamente para que seja possível interpretar os dados e replicar os métodos de cada estudo. Para a construção do presente ensaio, a base teórica se sustenta nos textos de Flávio Rezende, Jackson Alves de Aquino e Johanna Visser. Para construir a pesquisa, foi utilizada a abordagem qualitativa, interpretando os fenômenos estudados e relacionando os eventos. É possível concluir que o desenho de pesquisa é o elemento definidor da integração entre métodos, a partir da qual ferramentas oriundas de outras áreas do conhecimento podem ser utilizadas, como o Sistema $\mathrm{R}$ para estudos próprios das ciências jurídicas. Conclui-se, ainda, que o professor deve estimular a utilização de outros saberes para a construção do conhecimento científico de alto nível, a partir da construção de uma ponte dialógica entre ferramentas econométricas e a pesquisa jurídica de alto nível.

Palavras-chave: Sistema R. R-Studio. Pluralismo metodológico. Jurimetria.

\section{INTRODUÇÃO}

A pesquisa acadêmica se reveste de um questionamento fundamental, realizado antes mesmo do desenvolvimento do estudo: a abordagem deve seguir a linha qualitativa, com base na interpretação de fenômenos; ou deve ser constituída pela análise quantitativa, baseada em padrões numéricos e mensuração?

Ambas as abordagens decorrem da mesma lógica inferencial, o que, de imediato, já confere sustentação à complementaridade, à pluralidade metodológica para a construção de inferências.

A inferência também se baseia na interpretação, ou seja, na compreensão sobre o sentido das ações e interações dos indivíduos sob seus próprios pontos de vista. Trata-se de uma questão semântica, essencial para a formulação de hipóteses.

Entretanto, as pesquisas jurídicas se utilizam, tradicionalmente, da abordagem unicamente qualitativa, o

\footnotetext{
${ }^{1}$ Doutoranda em Ciência Política pela Universidade Federal de Pernambuco - PPGCP/UFPE (Conceito 7, bolsista CAPES). Professora da Universidade de Fortaleza. Pesquisadora do Grupo Epistemologia e Método na Ciência Política Comparada (Cnpq/UFPE).Pesquisadora do Multidoor Courthouse System. E-mail: mariana.dionisio@gmail.com
} 
que nem sempre é considerado científico na medida em que a mensuração, a replicabilidade do método e a precisão quanto aos dados pode restar comprometida.

Não há um mecanismo que possa determinar com exatidão como alcançar o equilíbrio entre o uso de indicadores quantitativos e qualitativos, o que leva a presumir que os pesquisadores estão sujeitos à dados imprecisos e erros de estimação, devendo assumir o compromisso de evidenciar a incerteza ínsita nos dados, quando houver.

O principal é utilizar a medida mais apropriada para os fins teóricos aos quais o pesquisador se propõe. Nesse sentido, ferramentas próprias de outras ciências podem ser extremamente úteis, porque podem realizar uma análise mais acurada do fenômeno estudado, fornecendo outras perspectivas sobre o conhecimento.

O presente texto propõe a relação possível entre a construção de desenhos de pesquisa que considerem a resolução de problemas jurídicos de mensuração de fenômenos a partir do uso do Sistema R, de base econométrica.

O estudo se divide em quatro tópicos: abordagem da pesquisa quali-quanti, refletindo a necessária complementaridade para o estudo das ciências jurídicas; o uso do Sistema R para a análise de dados em pesquisas jurídicas; jurimetria e suas aplicações; e a relevância da inserção de ferramentas tecnológicas no ensino superior, destacando-se o papel do professor no equilíbrio entre métodos tradicionais de ensino e conhecimentos oriundos de outras áreas.

O Sistema R é um software típico do uso estatístico porque sua execução depende de comandos e funções oriundas de equações econométricas, entretanto, é fortemente aplicável em áreas específicas de estudo diferentes da originária, como nas ciências jurídicas.

Essa transição quanto à utilização de uma ferramenta estatística para o estudo jurídico, tradicionalmente dialético, pode ser realizada de forma transversal, uma vez que não se propõe a substituir a análise qualitativa dos fenômenos políticos, jurídicos e sociais, mas sim, ampliar a consistência das inferências extraídas de hipóteses formuladas pelo pesquisador, propondo testes de hipóteses e análise de dados, sejam eles constituídos por padrões numéricos ou nominais.

O Sistema R possui um pacote de dados para funções específicas, o que não impede a inclusão de novos pacotes ou alteração de funções para melhor adequação às necessidades do pesquisador ou do estudo. Esse sistema possui fortes recursos de programação orientada por objetos, comparativamente à maioria das linguagens de computação estatística. Tal característica pode tornar menos simples a tarefa de consolidar o conhecimento sobre os comandos, mas não inviabiliza a análise dos bancos de dados.

Aqui, se propõe a utilização da modalidade $R$-Studio, que faz parte do extenso rol de possibilidades do Sistema $\mathrm{R}$, mas que parece se adequar melhor às demandas dos estudos voltados à análise de fenômenos sociais, 
políticos e jurídicos.

A presente pesquisa foi realizada com base em estudo descritivo-analítico, com aportes teóricos fundados, principalmente, em textos de Flávio da Cunha Rezende, Thomas Gschwend, Frank Schimmelfennig, para a construção das ideias de integração metodológica, e Jackson Aquino para a descrição da utilização do software Sistema R.

A pesquisa foi desenvolvida com o intuito de obter não apenas o maior aproveitamento quanto ao assunto abordado, mas, principalmente, a produção científica de maior alcance e produtividade para o ambiente acadêmico, especialmente para as ciências jurídicas.

\section{PESQUISA QUALI-QUANTI: A NECESSÁRIA COMPLEMENTARIDADE PARA O ESTUDO DAS CIÊNCIAS JURÍDICAS}

Tradicionalmente, é possível verificar o predomínio da abordagem qualitativa sobre a quantitativa nos estudos acadêmicos da área jurídica, especialmente pela necessidade de interpretar a realidade e os fenômenos sociais, a partir de elementos discursivos cuja defesa, não raro, se funda na argumentação dialética. Entretanto, é necessário repensar esse modelo, adotando, quando possível, configurações numéricas capazes de sustentar e conferir maior acuidade às análises jurídicas.

A distinção entre pesquisa qualitativa e quantitativa é frequentemente realizada de maneira inadequada. Algumas soluções para os problemas de pesquisa são comuns nas duas abordagens, e outras são transversais aos dois tipos. (Gschwend; Schimmelfennig, 2011).

Enquanto a pesquisa qualitativa se importa com abordagens pautadas no método discursivo, com informações mais aprofundadas sobre os temas estudados, a pesquisa quantitativa se baseia em mensurações numéricas, dados exatos e métodos estatísticos replicáveis. Esse modelo torna possível a iniciativa de testar hipóteses jurídicas em outras realidades sociais; o que ocorre, por exemplo, na análise legislativa comparada entre países.

Um dos temas mais recorrentes no que se refere à produção científica é o questionamento sobre a metodologia utilizada na pesquisa: seria mais adequada a abordagem qualitativa ou quantitativa? Há limites e possibilidades relativos à correlação entre os métodos quantitativos e qualitativos (Rezende, 2011), o que deve ser analisado pelo pesquisador não apenas a partir da natureza da pesquisa, mas fundamentalmente a partir do desenho de pesquisa e dos dados disponíveis.

Um desenho de pesquisa consiste em um plano que especifica a forma como o pesquisador pretende desenvolver o projeto de pesquisa e, particularmente, como as evidências localizadas serão utilizadas para responder a pergunta de pesquisa. 
Em um nível mais genérico, o problema de pesquisa, que descende do desenho de pesquisa, pode ser concebido como um diálogo entre teoria e dados. Pesquisadores formulam uma teoria, analisam os dados e os testam, reformulando sua teoria à luz das evidências empíricas, direcionando os testes à reformulação da teoria a partir dos dados.

Pesquisadores fazem observações, desenvolvem uma teoria para explicá-las, usam dados adicionais para testar sua teoria e, possivelmente, tornam a reformulá-las. Mas um projeto de pesquisa individual não necessariamente transcorre esse ciclo.

A ciência é um empreendimento coletivo, ou seja; alguns projetos de pesquisa vão focar no teste de hipótese, outros na explicação sobre observações específicas e geração de novas hipóteses (mas todos, deve-se ressaltar, constituem um diálogo entre teoria e dados). (Gschwend; Schimmelfennig, 2011).

O pesquisador deve verificar se há conexão entre os desenhos de pesquisa com abordagem qualitativa e quantitativa, na medida em que o desenho será o elemento que impulsionará todo o desenvolvimento do estudo.

Sobre ambos os desenhos de pesquisa pesam discussões quanto à validade e eficiência de seu uso, especialmente pela resistência apresentada por certas áreas do conhecimento em utilizar um ou outro método, considerando-os absolutamente antagônicos e substituindo a complementaridade dos métodos pelo mero maniqueísmo acadêmico. Mas ambos decorrem da mesma lógica de inferência.

$\mathrm{Na}$ investigação qualitativa os desenhos de pesquisa nem sempre são explícitos (alguns são imprecisos ao ponto de dificultar a compreensão sobre suas hipóteses causais); o que também pode ocorrer na pesquisa de natureza quantitativa.

Entretanto, as consequências decorrentes dessa imprecisão são diferentes nas modalidades citadas; enquanto no método qualitativo a falta de definição quanto ao desenho de pesquisa pode gerar apenas a falta de estimações, a falta de precisão no desenho de pesquisa quantitativo pode gerar conclusões incoerentes. (Keohane; King; Verba, 1994).

Os estudos quantitativos dispõem de medidas mais precisas por serem numéricas, o que não significa dizer que são necessariamente infalíveis; razão pela qual é fundamental testar as hipóteses formuladas.

Nesse sentido, o Sistema R se adequa como ferramenta de apoio aos testes, fortalecendo ou refutando as inferências elaboradas pelo pesquisador.

Esse esforço de testar as hipóteses por meio de uma ferramenta complementar garante não apenas a o aperfeiçoamento do conhecimento empírico sobre o fenômeno social ou jurídico estudado, mas também rejeita hipóteses empiricamente não confirmadas, dando maior refinamento à teoria formulada. A análise de dados não é um método de pesquisa, mas sim, estratégia que pode ser inserida em quaisquer abordagens, sejam quantitativas ou qualitativas. 
Para o ensino jurídico, é fundamental que haja, por parte do professor, a iniciativa de abordar o teste de hipóteses como meio para fortalecer a pesquisa nas diversas searas do Direito, especialmente nos estudos voltados à análise de dados que verifiquem a eficiência legislativa, linha de orientação jurisprudencial de tribunais estaduais ou superiores, ou que apresentem evidências sobre a atuação institucional do Ministério Público, Defensoria Pública etc.

É necessário refletir sobre a possível integração entre métodos, uma vez que a complementaridade entre as abordagens, chamada por pluralismo metodológico, pode ser viabilizada a partir da mera adoção de ferramentas para testar hipóteses ou evidências empíricas, conferindo maior sustentação às teorias propostas pelo pesquisador. Trata-se de uma preocupação inferencial, absolutamente elementar para o avanço disciplinar. (Rezende, 2014).

Essa integração pode contar com a contribuição de novos saberes, oriundos de outras áreas do conhecimento, o que torna frutífera a relação entre a pesquisa e o uso, aqui proposto, de ferramentas estatísticas para o alcance de resultados nas ciências jurídicas.

\section{O SISTEMA R PARA A ANÁLISE DE DADOS EM PESQUISAS JURÍDICAS}

O Sistema R consiste em um software avançado de estatística compatível com sistemas operacionais como Microsoft Windows e Mac OS X, que, a partir de comandos básicos e funções derivadas, seleciona bases de dados disponíveis em sistemas cadastrados (inclusive internet, bastando, para isso, carregar os dados no próprio sistema), e pode construir uma robusta análise estatística desses dados, sem que seja necessário comprar módulos adicionais para executar funções extras.

Com esse sistema, é possível criar e manter disponíveis, na área de trabalho do computador de acesso, vários tipos de objetos e fórmulas (scripts), o que permite maior flexibilidade e rapidez na execução dos comandos, inclusive para bancos de dados muito grandes (Aquino, 2014).

O sistema, que utiliza linguagem de programação para o cálculo estatístico, se divide em vários ambientes de desenvolvimento integrado, como R-Studio, R-Commander, R-Shell, R-Python etc. Todos se destinam à execução de funções estatísticas e econométricas, mas sua utilização dependerá da necessidade e ao estilo de trabalho do pesquisador, visto que utiliza plataformas visuais diferentes.

Para a área jurídica, o Sistema R-Studio se apresenta como o mais adequado simplesmente por possuir comandos mais simples e permitir que as fórmulas sejam salvas de maneira mais acessível na área de trabalho, o que facilita a pesquisa de profissionais que, em sua maioria, não possuem familiaridade com regras estatísticas, como é o caso dos pesquisadores das ciências jurídicas.

Para descrever o impacto causado pelo quantitativo de processos, sejam de matéria tipicamente federal 
ou da competência da justiça estadual, durante períodos específicos, o Sistema R se apresenta como uma alternativa viável na medida em que pode fornecer gráficos demonstrativos, análises percentuais e avaliação de impacto, simultaneamente e a partir de uma única base de dados (seja ela grande ou pequena).

Trata-se de uma possibilidade mais barata para o Poder Judiciário (tanto que já é utilizado para as análises de dados coletados pelo Conselho Nacional de Justiça - CNJ) e para o uso universitário, visto que o software pode ser adquirido por instituições de ensino superior, por valores mais baratos que outros softwares avançados similares como o Stata (Data Analysis and Statistical Software) o SAS (Statistical Analysis Software) ou o SPSS (Statistical Package for Social Science), por meio de uma licença acadêmica cuja renovação é anual.

Em pesquisas que envolvem a análise quantitativa da atuação de tribunais estaduais, por exemplo, o Sistema $\mathrm{R}$ pode, com a mesma base de dados (como número total de recursos recepcionados, número de servidores, produtividade em relação ao orçamento anual, acompanhamento de cumprimento de decisão etc.), produzir modelação linear e não linear, testes estatísticos clássicos, análise de séries temporais (time-series analysis - o que é extremamente útil quando se utiliza periodização específica para análise de resultados; além de fazer classificação, agrupamento e elaboração de gráficos, simultaneamente (Aquino, 2014).

Segue o padrão de tela do Sistema R, aqui representado na versão R-Studio:

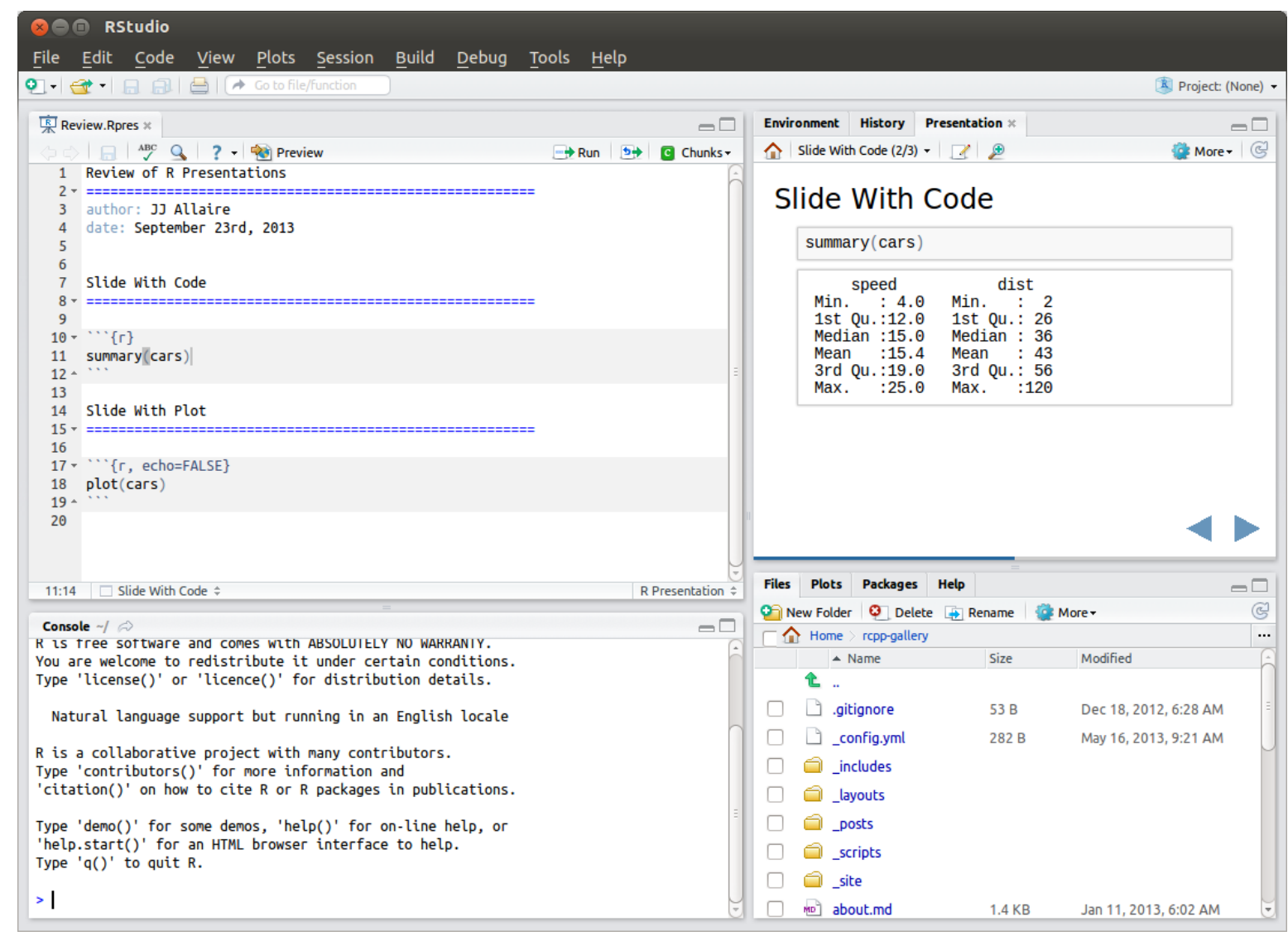

Fonte: https://www.rstudio.com/ 
A referida imagem evidencia as quatro telas simultâneas, que correspondem, em sentido horário, ao armazenamento das fórmulas completas ( $R$ script) que podem ser salvas em formato de comandos na área de trabalho ao final da execução; ao desenvolvimento dos cálculos estatísticos ( $R$ environment), em que os comandos digitados na primeira área serão convertidos numericamente; à tela de visualização de gráficos e tabelas (Graphical output), para demonstração gráfica dos cálculos realizados e melhor visualização de respostas; e, finalmente, à tela de inscrição dos comandos ( $R$ console) .

A manipulação do Sistema $\mathrm{R}$ depende de empenho e repetição. $\mathrm{O}$ pesquisador deve tentar repetir comandos, alterando opções, para que se sinta mais familiarizado com a ferramenta, já tradicional no uso de estudos econométricos.

Há uma extensa bibliografia auxiliar disponível em acesso público para que o pesquisador iniciante tenha menor dificuldade para assimilar esse novo conhecimento. Entretanto, quando ainda não há familiaridade com determinados comandos, o pesquisador pode contar com o próprio Sistema R para localizar as fórmulas com os comandos (scripts) em outras plataformas, visto que a maioria delas está disponibilizada, por outros usuários, na internet. (Aquino, 2014).

Importa ressaltar, ainda, que o Sistema $\mathrm{R}$ não se limita a realizar os testes com base numérica, uma vez que sua plataforma admite a transcrição de bases de dados nominais (o que atesta, mais uma vez, a necessidade de interpretação dos dados pelo pesquisador, que só pode fazê-lo se possui uma formação adequada em abordagem qualitativa - confirmando a proposta da complementaridade defendida nos parágrafos iniciais do presente estudo).

O R-Studio é uma modalidade do software R, que também foi formatado em um ambiente de desenvolvimento integrado para o sistema e que também utiliza linguagem de programação para gráficos e cálculos estatísticos (Aquino, 2014), mas que vem ganhando espaço no campo acadêmico por ser dotado de características como menor complexidade na instalação, custo de aquisição mais baixo (algumas versões gratuitas já compatíveis com Microsoft Windows e Mac OS X estão disponibilizadas na internet), pacotes adicionais acessíveis pela internet e comandos alteráveis de acordo com a necessidade do pesquisador.

Além disso, há a vantagem adicional de quatro consoles (telas com edição de texto e telas com exibição de gráficos, cálculos estatísticos e tabelas) simultâneos, que permitem melhor visualização entre os comandos digitados e o resultado.

Para estudos que envolvem a análise de jurisprudência, o R-Studio evidencia possibilidades como: visualização de linearidade ou orientação jurisprudencial para cada tribunal estadual sobre determinado tema a partir de bases de dados nacionais, construção de tabelas e gráficos para vários temas diferentes simultaneamente, análise time series com períodos extensos (períodos acima de dez anos, inclusive) e construção de gráficos 
pontuados para identificação dos tribunais que mais seguem determinada orientação (as orientações devem ser inseridas nominalmente pelo pesquisador).

\section{JURIMETRIA}

A jurimetria traduz a aplicação de métodos quantitativos que descendem da análise estatística ao Direito, uma vez que propicia uma visão probabilística de fenômenos de interação no contexto jurídico.

Essa utilização não é recente, mas ainda é pouco conhecida em países de tradição jurídica romanogermânica, como o Brasil, pelo simples fato de que há um distanciamento natural entre pesquisadores de ciências de campos científicos diferentes; o que também se aplica aos pesquisadores voltados à análise das ciências jurídicas, cuja abordagem quantitativa dos estudos não é uma constante.

O estudo da jurimetria descende da década de 1950, e foi difundida nos Estados Unidos por Lee Loevinger, advogado que no artigo Jurimetrics: The Next Step Forward publicado na revista Minnesota Law Review, descreveu possibilidades para o uso de métodos quantitativos aplicáveis ao direito, com a finalidade de documentar e racionalizar informações. É um estudo desenvolvido por meio de processos de análise, pesquisa e gestão de dados que permite ao pesquisador uma visão sistemática das decisões jurídicas. (Ramírez; Díaz; Fernández, 2016).

A jurimetria fornece uma perspectiva sistemática dos fatores que influenciam ou que exercem algum papel na tomada de decisões pelo magistrado, na medida em que contribui para definir, com suporte em elementos quantitativos, padrões de comportamento legal.

Com o auxílio de padrões jurimétricos de investigação, é possível identificar e conferir maior transparência e previsibilidade às decisões jurídicas. A investigação jurisdicional também pode aumentar a legitimidade do sistema jurídico, exercendo um papel de supervisão e monitoramento sobre as partes e procedimentos envolvidos. (Visser, 2006; Ramírez; Díaz; Fernández, 2016).

Com o auxílio de profissionais de outras áreas (Matemática, Estatística, Economia), coletam-se dados para revisão, análise e em seguida verificação dos resultados, comparando-os com outros estudos e com as perspectivas de órgãos de accountabilityjudicial, como o Conselho Nacional de Justiça.

A partir da análise econométrica, é possível identificar as maiores motivações para o acesso ao Judiciário (o que, por sua vez, serve para distinguir as ações mais recorrentes); promover a maior aproximação entre a realidade social e o universo jurídico; detectar padrões de resolução aplicados a cada caso concreto; contribuir para a melhora da prestação jurisdicional à sociedade; e construir demonstrativos e grandes bancos de dados com as informações coletadas para auxiliar em pesquisas acadêmicas.

É possível, ainda, perceber os efeitos das decisões sobre o jurisdicionado e o impacto no sistema de 
organização do Poder Judiciário, auxiliando os órgãos de controle na fiscalização e na construção de metas. (Visser, 2006).

A previsibilidade no meio jurídico se baseia em pesquisas large- $N$, ou seja, em bancos de dados com amostras numericamente expressivas e quantitativamente consideráveis.

Trata-se de uma acurada análise de metadados que podem auxiliar na identificação de modelos semelhantes de conduta. No contexto das decisões judiciais, há dezenas de milhares de processos com características similares, o que sugere a inegável possibilidade de uma verificação de padrão de comportamento dos tribunais e dos julgadores.

Como exemplo, há o polêmico IV Relatório Supremo em Números: o Supremo e o Ministério Público, assinado por Joaquim Falcão, Alexandre de Moraes, Michael Mohallem e Ivar Alberto Hartmann e publicado pela Fundação Getúlio Vargas - FVG, que, a partir da jurimetria, expõe possibilidades de padrão nos julgados na esfera do Supremo Tribunal Federal.

O relatório, com base estatística, apresenta análises que identificam um perfil de atuação do Ministério Público no Supremo Tribunal Federal, incluindo a taxa de sucesso com suporte em pesquisa empírica.

A pesquisa realizada utilizou como bases o Projeto Supremo em Números - projeto de pesquisa do Centro de Justiça e Sociedade (CJUS), da Escola de Direito da Fundação Getulio Vargas (FGV DIREITO RIO), no Rio de Janeiro; além de macroanálises de todos os processos do Supremo Tribunal Federal (no total, 40.635 processos), com periodização entre 1988 e 2013.

A despeito das críticas, o referido relatório apenas desenvolve o padrão econométrico já adotado pelos relatórios Justiça em Números realizados pelo Conselho Nacional de Justiça - CNJ desde 2008.

A iniciativa revela um campo de pesquisa profícuo para as ciências jurídicas, especialmente para a verificação de indicadores legais.

Com pesquisas empíricas, é possível estabelecer o cumprimento de metas, perceber quais tribunais cumprem tais metas e delimitar as razões para o eventual descumprimento, verificar o tipo de ação mais recorrente em cada instância, o volume recursal e os tipos de tese de defesa utilizados recorrentemente em casos semelhantes, fortalecer as teses sobre incidentes de demandas repetitivas como inovação do Código de Processo Civil, reafirmar valores propostos nas legislações específicas por meio da análise de resultados, utilização racional dos dados digitalizados pelos processos eletrônicos (que, por si, já criam um amplo banco de dados para consulta e criação de padrões) etc.

Há uma série de consequências interessantes para a pesquisa jurídica e para a aproximação entre as decisões e a realidade dos fatos. 


\section{INCLUSÃO DE FERRAMENTAS TECNOLÓGICAS NO ENSINO SUPERIOR}

É possível perceber que o Sistema $\mathrm{R}$ e as análises da jurimetria podem se adequar não apenas às pesquisas em nível de pós-graduação, dada sua compatibilidade com outros conhecimentos, mas à prática jurídica vivenciada no cotidiano forense e à pesquisa de alto nível.

Assim, verifica-se a necessidade de incluir tais estudos ainda na academia, propiciando a maior difusão das técnicas e a profusão do conhecimento na universidade.

A inclusão de ferramentas tecnológicas no ensino superior é uma tendência que deve ser estimulada continuamente pelo professor, incentivando pesquisadores a adotarem a pluralidade de possibilidades e estratégias possíveis para a construção de inferências válidas e realização de testes de hipóteses que fortaleçam a pesquisa.

A docência no ensino superior se relaciona com inovações e dinâmicas modernas de aprendizado, de forma complementar às metodologias tradicionais de ensino. Essa correlação tende a facilitar o processo de ensino e aprendizagem, desde que o professor esteja familiarizado com as possibilidades oferecidas por cada ferramenta.

Essa é a razão primordial para o desenvolvimento de estudos como o que aqui se apresenta: demonstrar que mecanismos típicos de outras áreas do conhecimento podem ser úteis ao ensino e à pesquisa jurídica, desde que haja disponibilidade e interesse dos pesquisadores em aprender e consolidar outros saberes.

A tecnologia, por si, não garante a eficiência das práticas de ensino, assim como os dados, sozinhos, não são capazes de responder aos problemas de pesquisa formulados. Deve haver uma contínua integração entre curiosidade, abertura científica e pluralidade metodológica para que as ciências possam intercambiar conhecimentos fundamentais.

Para o ensino superior na área jurídica ou para as demais, resta ao professor / pesquisador ampliar conceitos e perspectivas a partir da admissão de uma relação harmônica entre saberes diversos.

Não que se trate de um imperativo tecnológico, longe disso. Trata-se de uma valiosa oportunidade para que pesquisadores trabalhem coletivamente, reunindo ideias, conhecimentos e experiências, nas mais diversas áreas, para um fim comum: a produção de pesquisas de alto nível e de inconteste relevância para a sociedade.

\section{CONCLUSÃO}

Há um enorme potencial dessa ferramenta, seja pela modalidade R-Studio ou quaisquer das possibilidades fornecidas pelo Sistema $\mathrm{R}$, no apoio à pesquisa jurídica, que deve ser difundida especialmente durante a vivência universitária. 
Conforme ressaltado anteriormente, a tradicional pesquisa qualitativa presente da área jurídica é fundamental para a interpretação e consequente compreensão de fenômenos políticos, jurídicos e sociais; mas deve se aliar, quando cabível, ao empirismo, aos testes de hipóteses e, principalmente, à abordagem quantitativa, para que as experiências possam ser replicadas e para que seja possível conferir maior precisão às inferências extraídas de cada estudo.

Na prática docente, o estímulo à utilização de diferentes ferramentas para a pesquisa pode contribuir de forma significativa para a evolução das pesquisas de alto nível. É imprescindível que professores e alunos se mantenham atentos aos métodos herdados de outras áreas do conhecimento científico, ampliando a perspectiva da pesquisa em si.

O professor, como condutor desses saberes, deve promover a aproximação entre pesquisadores (em nível de graduação ou pós-graduação) e outros métodos de pesquisa, para que seja possível fazer uma escolha fundamentada e racionalizada sobre qual método é mais adequado para cada tipo de estudo, reduzindo a exclusão de possibilidades e ampliando saberes.

Cabe ao professor a habilidade de integrar métodos e estratégias para que a pesquisa científica gere inferências válidas, e a contribuição de ferramentas que obedecem padrões econométricos pode ser extremamente útil e oportuna, principalmente no que se refere ao teste de hipóteses.

O teste de hipóteses, que pode ocorrer em qualquer área do saber, se constitui em um método de inferência estatística que utiliza dados de um estudo científico com o objetivo de determinar se há indícios suficientes em uma amostra capaz de identificar se a condição observada para um indivíduo é também observada para uma população, como um mecanismo de generalização possível e bastante útil para a construção do conhecimento.

Conhecer a realidade a partir da utilização de métodos e ferramentas estatísticas pode ser um caminho útil para sua transformação.

Analisar o efeito das decisões judiciais para o jurisdicionado em curto e longo prazo, possibilitar condições de verificação do perfil do julgador, inovar os julgados com a utilização de ferramentas de inteligência artificial para saber quais teses são as mais aceitas em determinados casos, construir padrões de decisão etc., tudo isso serve para conferir previsibilidade; característica fundamental nas ciências jurídicas.

A construção desse conhecimento é um processo colaborativo, razão pela qual se constata ser plenamente possível a construção uma ponte dialógica e didática entre a pesquisa jurídica de alto nível e a utilização de métodos com base econométrica, ampliando-se assim a contribuição de outras ciências à esfera jurídica. 


\title{
THE USE OF THE R-STUDIO SYSTEM AND JURIMETRICS AS COMPLEMENTARY TOOLS FOR THE LEGAL RESEARCH
}

\begin{abstract}
The present research aims to answer the following question: Is it possible to build a dialogical and didactic bridge between high level legal research and the use of econometric methods? The main idea is the use of R-Studio software, typical of statistical studies, as a tool to support legal research based on data analysis, which can already be done by jurimetrics and should be widely disseminated by teaching practices. The complementarity between quantitative and qualitative research is essential, precisely so that it is possible to read the data and replicate the methods of each study. This essay have theoretical basis on texts by Flávio Rezende, Jackson Alves de Aquino and Johanna Visser. To construct the research, the qualitative approach was used, interpreting the studied phenomena and relating the events. It is possible to conclude that the research design is the defining element of the integration between methods, from which tools from other areas of knowledge can be used, such as System R for studies of the legal sciences. It is also concluded that the teacher should stimulate the use of other knowledge for the construction of high level scientific knowledge, from the construction of a dialogical bridge between econometric tools and high level legal research.
\end{abstract}

Keywords: R System. R-Studio. Metodological pluralism. Jurimetrics.

\section{REFERENCIAS}

AQUINO, Jakson Alves de. R para cientistas sociais. Ilhéus: Editora da UESC, 2014.

GSCHWEND, Thomas; SCHIMMELFENNIG, Frank. Research design in political science: how to practice what they preach. England: British Library, 2011.

KELLSTEDT, Paul M.; WHITTEN, Guy D. The fundamentals of political science research. Cambridge: University Press, 2009.

KING, Gary; KEOHANE, Robert; VERBA, Sidney. Designing social inquiry. New Jersey: Princenton University Press, 1994.

RAMÍREZ, René Acosta; DÍAZ, Yadirka Verdecia; FERNÁNDEZ, Yarina Amoroso. Jurimetría: una opción para la sociedad. In: Serie Cientifica de la Universidad de las Ciencias Informáticas, vol. 9, n. 4, 2016, pp. 1-10.

REZENDE, Flávio da Cunha. A "nova metodologia qualitativa" e as condições essenciais de demarcação entre desenhos de pesquisa na ciência política comparada. In: Revista Política Hoje, vol. 20, n. 1, 2011, pp. 218 - 252.

Fronteiras de integração entre métodos quantitativos e qualitativos na ciência política comparada. In: Revista Teoria e sociedade, n. 22, 2014, pp. 40 - 74.

VISSER, Johanna. Jurimetrics, safety and security. In: International Review of Law, Computers and Technology, vol. 20, n. 1/2, 2006, pp. 123 - 145.

HARTMANN, Ivar A.; FALCÃO, Joaquim; MORAES, Alexandre de (Org.). IV Relatório Supremo em 
Números: o Supremo e o Ministério Público. Rio de Janeiro: Escola de Direito do Rio de Janeiro da Fundação Getulio Vargas, 2015.

Trabalho enviado em 18 de junho de 2017.

Aceito em 09 de setembro de 2017. 